\title{
Theoretical determination of the radiation force for a spherical particle illuminated by a focused laser beam
}

\author{
R.R. Dorizzi $i^{1}$ and Z. Ulanowski ${ }^{2}$ \\ ${ }^{1}$ Department of Physics, Astronomy and Mathematics, University of Hertfordshire, \\ College Lane, Hatfield, Herts AL10 9AB, UK \\ ${ }^{2}$ Science and technology Research Institute, University of Hertfordshire, College Lane, Hatfield, Herts \\ $A L 109 A B, U K$ \\ tel: +44 (0) 1707-286124, fax : +44 (0) 1707-284256, e-mail: r.r.dorizzi@herts.ac.uk
}

\begin{abstract}
Trapping forces on dielectric spheres in single beam laser tweezers are computed. A focused beam description based on an exact solution of Maxwell's equations is compared to the 5th order Gaussian beam approximation due to Barton and Alexander. Forces on water droplets suspended in air and on polystyrene spheres suspended in water, exerted by beams focused to varying degree, are calculated. It is demonstrated that the 5th order approximation is accurate for almost paraxial beams (numerical aperture NA < 0.25), as compared to the exact treatment. However, for strongly focused beams the 5th order approximation breaks down. Thus it is established that an accurate beam description is vital for modeling optical traps, since, in order to hold a particle effectively in a single beam trap, a strongly focused beam is required.
\end{abstract}

Key Words: Electromagnetic Field, Focused Gaussian Laser Beam, Optical Trapping Forces

\section{Introduction}

Starting with the experiments of Ashkin in 1970 [1] it became possible to use laser beams to trap a variety of particles, including living cells [2], organelles within cells [3] and even larger objects like the giant amoeba [4]. There are various types of such "laser tweezers" in use, including single and the multi beam ones. It is the aim of this work to present a theoretical model of single beam laser tweezers which uses a computational method developed by Barton et al. [5]. Since the models presented in [5] and Moine and Stout [6] are based on the electromagnetic (EM) field derived from the 5th order Gaussian beam approximation [7], the accuracy of the results obtained using these models is uncertain. Hence in order to test the accuracy of the results, we replace the to 5th order approximation by EM fields that are exact solutions to Maxwell's equations [8]. Gouesbet et al. [9] have presented a localized interpretation to compute the coefficients in the generalized Lorenz-Mie theory. Mazolli et al. [10] presented another model, which is based on the Debye-type integral representation of the laser beam as a superposition of plane EM waves. The main difference between Mazolli et al. [10] and Barton et al. [5] as well as the model presented here is that Mazolli et al. [10] take truncations of the beam by the focusing lens into account. This is not the case in the model of Barton et al. [5] and the model presented here, nor are lens aberrations taken into account.

\section{Theory and results}

Lorenz [11] and Mie [12] formulated a theory with which the EM fields inside and outside a sphere can be calculated, when a plane incident wave is scattered by the sphere. Barton et al. [5] have used this theory, generalised to an arbitrary incident field, to compute radiation forces and torques exerted on a spherical particle. They calculated the Mie scattering coefficients by integrating over the radial part of the beam fields. Ulanowski and Jones [13] have written a set of computer programs to model the trapping forces as derived by Barton et al. [5] for real relative refractive indices $n$, which is adequate for calculating the trapping forces for nonabsorbing particles. Here the programs are used with Barton's 5th order beam approximation, and the "exact" solutions (Eqs. 1 and 2) to Maxwell's equations presented by Dorizzi [8]. 
These vector solutions were obtained using the method given by Bohren and Huffman [14] of constructing vector functions $\mathbf{M}$ and $\mathbf{N}$ from a scalar function; here the order 01 from the family of scalar, non-paraxial beam solutions derived by Ulanowski and Ludlow [15]. Dorizzi [8] formed a superposition of the $\mathbf{M}$ and $\mathbf{N}$ functions which lead to the EM field of a beam propagating along the $z$ axis of a Cartesian coordinate system $(x, y, z)$ :

$$
\begin{gathered}
\mathbf{E}_{01}(x, y, z)=\frac{E_{0}(z-i d) e^{-k d} j_{2}(k R)}{k R^{4}}\left(\begin{array}{c}
i x y\left(5-\frac{k R j_{1}(k R)}{j_{2}(k R)}\right) \\
k R^{2}\left(\frac{R j_{1}(k R)}{k(z-i d) j_{2}(k R)}-(z-i d)\right)+i\left(4 R^{2}-\left(R^{2}-y^{2}\right)\left(5-\frac{k R j_{1}(k R)}{j_{2}(k R)}\right)\right) \\
\left.y\left(k R^{2}-i\left(\frac{R^{2}}{z-i d}-(z-i d)\left(5-\frac{k R j_{1}(k R)}{j_{2}(k R)}\right)\right)\right)\right) \\
\mathbf{H}_{01}(x, y, z)=\frac{-i E_{0} e^{-k d} j_{2}(k R)(z-i d)}{k R^{4}}
\end{array}\right. \\
\sqrt{\frac{\varepsilon}{\mu_{0}}}\left(\begin{array}{c}
\left.-i k R^{2}\left(\frac{R j_{1}(k R)}{k(z-i d) j_{2}(k R)}-(z-i d)\right)+4 R^{2}-\left(R^{2}-x^{2}\right)\left(5-\frac{k R j_{1}(k R)}{j_{2}(k R)}\right)\right) \\
x y\left(5-\frac{k R j_{1}(k R)}{j_{2}(k R)}\right) \\
x\left(-i k R^{2}-\frac{R^{2}}{z-i d}+(z-i d)\left(5-\frac{k R j_{1}(k R)}{j_{2}(k R)}\right)\right)
\end{array}\right) .
\end{gathered}
$$

This EM field is linearly polarized along the $y$ axis in the paraxial limit and also satisfies the Richards and Wolf [16] far-field boundary conditions. In the above expressions, $k$ is the wave number, $d=k w_{0}^{2} / 2$ is the Rayleigh range, where $w_{0}$ is the beam waist radius, $\varepsilon_{0}$ and $\mu_{0}$ are the free space permittivity and permeability, respectively, $\varepsilon$ is the absolute permittivity and $R=\left[x^{2}+y^{2}+(z-i d)^{2}\right]^{1 / 2}$. The spherical Bessel functions in the above expressions are given in explicit form by Eqs. 3 and 4 :

$$
j_{1}(k R)=\frac{\sin k R}{(k R)^{2}}-\frac{\cos k R}{k R}
$$

and

$j_{2}(k R)=\frac{3 \sin k R}{(k R)^{3}}-\frac{3 \cos k R}{(k R)^{2}}-\frac{\sin k R}{k R}$.

Barton et al. [7] used an expression for the beam power $P$, which was approximated to the 5th order Gaussian beam based on the irradiance normalized to unity at $(x=y=z=0)$. The corresponding expression for the "exact" treatment is

$$
P_{\text {norm }}=\frac{\pi E_{0}^{2} \sqrt{\varepsilon}}{4 k^{2} \sqrt{\mu_{0}}} \frac{p^{3}\left(e^{2 p}\left(8 p^{4}-12 p^{3}+13 p^{2}-9 p+3\right)+e^{-2 p}\left(p^{2}+3[p+1]\right)-2\left(p^{2}+3[1-p]\right)\right)}{\left.\left.e^{6}-4 p^{5}+9 p^{4}-13 p^{3}\right]+49 p^{2}-30 p+9\right)+e^{-2 p}\left(p^{2}+3[2 p+3]\right)+4 p\left[p^{3}+p^{2}+6\right]-14 p^{2}-18},
$$


where $p=k d$. In this work a lens diameter of 3 times the beam radius is assumed, resulting in a $98.9 \%$ power transmission. In the calculations the power transmission is taken to be $100 \%$, producing only a small error. Under paraxial assumptions the focal length $f$ of a focusing lens can be related to the beam parameters as $f=w \sqrt{k d / 2}$, where $w$ is beam radius [15]. Therefore in our case the numerical aperture of the focusing lens can be directly related to the beam parameters by $N A=3 / k w_{0}$. The square of the electric field amplitude at the focal point of the beam $(x=y=z=0)$ can be related to the normalized beam power $P_{\text {norm }}$ by rearranging Eq. 5 to give

$$
E_{0}^{2}=\frac{4 P_{\text {nоm }} k^{2} \sqrt{\mu_{0}}}{\pi \sqrt{\varepsilon}} \frac{e^{2 p}\left(4\left[p^{6}-4 p^{5}+9 p^{4}-13 p^{3}\right]+49 p^{2}-30 p+9\right)+e^{-2 p}\left(p^{2}+3[2 p+3]\right)+4 p\left[p^{3}+p^{2}+6\right]-14 p^{2}-18}{p^{3}\left(e^{2 p}\left(8 p^{4}-12 p^{3}+13 p^{2}-9 p+3\right)+e^{-2 p}\left(p^{2}+3[p+1]\right)-2\left(p^{2}+3[1-p]\right)\right)} .
$$

In the "exact" treatment, the expressions given in Eqs 1,2 and 6 are replacing the corresponding expressions of the 5th order Gaussian Beam approximation as given by Barton et al. [5] and so the trapping forces can be calculated using the same formulae. The acceleration exerted by a focused laser beam with $k d=74.6$, beam power $P=3.5 \mathrm{~mW}$, beam waist radius $w_{0}=1 \mu \mathrm{m}$ and wavelength $\lambda_{0}=514.5 \mathrm{~nm}$, on a spherical water droplet (refractive index $n=1.334$ [5]) of diameter $D=4.96 \mu \mathrm{m}$ and mass $m_{w}=6.39 \times 10^{-14} \mathrm{~kg}$ suspended in air, is calculated using the 5 th order Gaussian beam approximation and the "exact" treatment. Fig. 1 compares the acceleration $a_{z}=F_{z} / m_{w}$ calculated using both methods for sphere positions along the axis of propagation. However, the restoring acceleration is expressed in relation to the acceleration due to gravity $g$. The offset along the $x$ and $y$ axis is zero $\left(x_{0}=y_{0}=0\right)$.

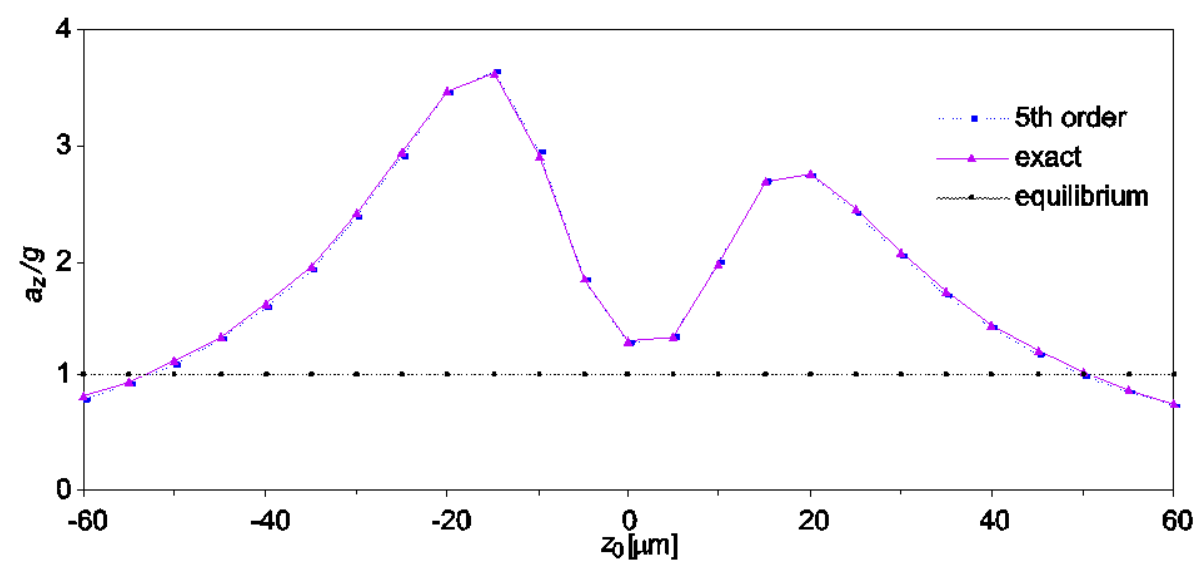

Fig. 1. Comparison of the restoring acceleration based on the 5th order Gaussian beam approximation (doted curve) and the "exact" treatment (solid curve), along the propagation axis for optical levitation of a water droplet in air, using a focused, linearly polarised laser beam, $D=4.96 \mu \mathrm{m}, \bar{n}=1.334$, wavelength $\lambda_{\square 0}=0.5145 \mu \mathrm{m}$, beam power $P=3.5 \mathrm{~mW}$ and beam waist radius $w_{0}=1 \mu \mathrm{m}$, corresponding to lens numerical aperture $\mathrm{NA} \approx 0.246$ or $k d=74.6$.

It can be seen from Fig. 1 that near the axial position $z_{0}=-50 \mu \mathrm{m}$ the water droplet is in an unstable equilibrium and near $z_{0}=50 \mu \mathrm{m}$ in a stable equilibrium, assuming that the force of gravity is directed towards negative $z$ values. The average RMS error between the two models of all the calculated points in Fig. 1 is $1.4 \%$. Since these restoring acceleration values are for an almost paraxial Gaussian beam $(k d=74.6)$, they are very useful to benchmark the restoring acceleration values based on the "exact" treatment. In the case where the restoring acceleration is calculated along the $x$ axis in the 5th order 
Gaussian beam approximation (along the direction of polarisation), it has to be compared with the restoring acceleration along the $y$ axis in the "exact" treatment, since the $y$ axis is the direction of polarisation for this case. Barton et al. [5] chose the stable equilibrium position $z_{0}=50 \mu \mathrm{m}$ above the focal point. The beam diameter is $2 w_{0} \approx 16.5 \mu \mathrm{m}$ at this point, which is greater than the droplet diameter. In order to calculate the restoring accelerations along the $x$ axis, $y_{0}=0$. In the "exact" treatment, as the direction of polarisation is the $y$ axis, the restoring acceleration along this axes is calculated with $x_{0}=0$. Fig. 2 compares the restoring acceleration for sphere positions along the direction of polarization $\left[a_{x}\right]$ and $\left[a_{y}\right]$ respectively, calculated using both methods. Fig. 3 compares the restoring acceleration for sphere positions along the axis perpendicular to the direction of polarisation $\left[a_{y}\right]$ and $\left[a_{x}\right]$ respectively, calculated using both models.

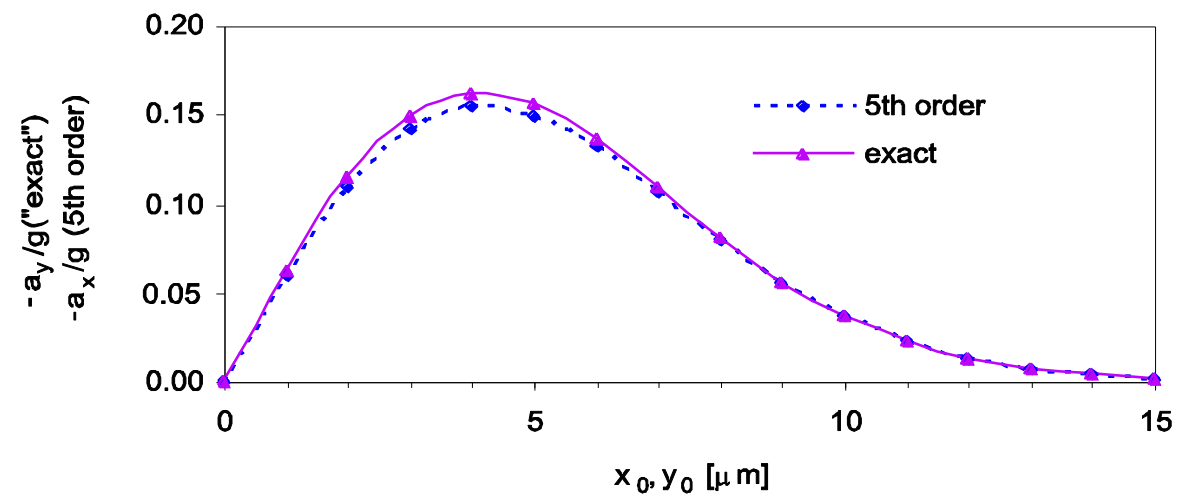

Fig. 2. Comparison of the restoring acceleration based on the 5th order Gaussian beam approximation (doted curve) and the "exact" treatment (solid curve), along the polarisation axis versus displacement along the polarisation axis for optical levitation of a water droplet in air, using a focused order 01 mode linearly polarised laser beam at a droplet propagation axis position of $z_{0}=50 \mu \mathrm{m}$ above the focal point $\left(x_{0}=y_{0}=0, z_{0}=50 \mu \mathrm{m}, \bar{n}=1.334, D=4.96 \mu \mathrm{m}\right.$, $\lambda_{0}=0.5145 \mu \mathrm{m}, w_{0}=1 \mu \mathrm{m}, k d=74.6$ and $\left.P=3.5 \mathrm{~mW}\right)$. 


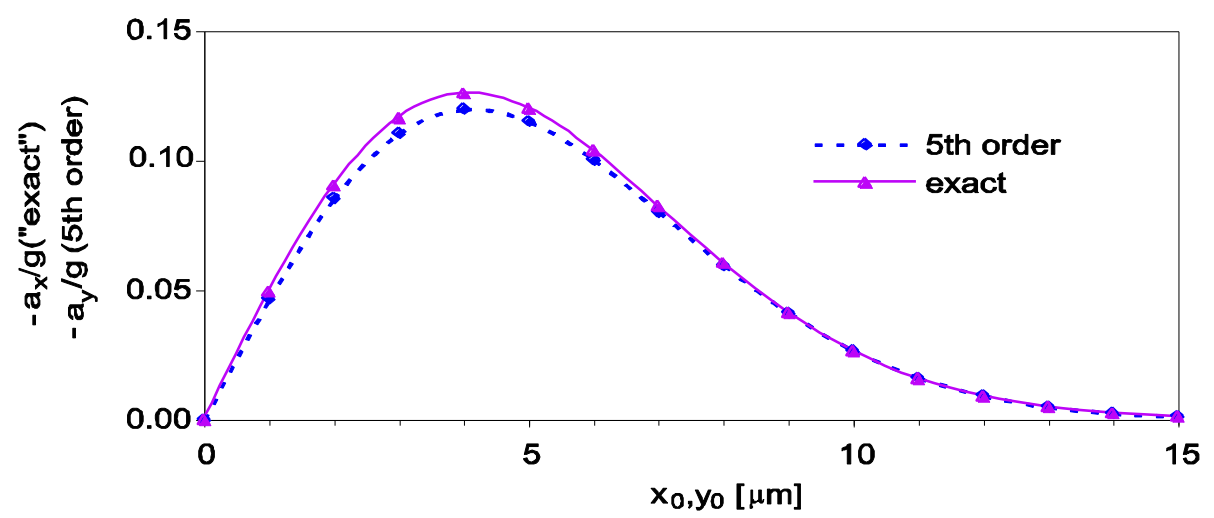

Fig. 3. Comparison of the restoring acceleration based on the 5th order Gaussian beam approximation (doted curve) and the "exact" treatment (solid curve), along the axis perpendicular to the direction of polarisation versus displacement along the axis perpendicular to the direction of polarisation axis for optical levitation of a water droplet in air, using a focused order 01 mode linearly polarised laser beam $\left(x_{0}=y_{0}=0 \mu \mathrm{m}, z_{0}=50 \mu \mathrm{m}, \bar{n}=1.334, D=4.96 \mu \mathrm{m}\right.$, wavelength $\lambda_{0}=0.5145 \mu \mathrm{m}, w_{0}=1 \mu \mathrm{m}, k d=74.6$ and $\left.P=3.5 \mathrm{~mW}\right)$.

By comparing Figs. 2 and 3 it can be seen, as expected, that the restoring acceleration along the polarisation axis of the beam is greater than the restoring acceleration along the axis perpendicular to the direction of polarisation. This is due to the fact that the Fresnel reflectance and transmittance coefficients are different for an incident EM wave polarised parallel and perpendicular to the incident plane. If the EM field is polarised parallel to the incident plane, more radiation is transmitted. Since optical trapping is dependent on the amount of refracted radiation, the trapping forces are larger for particle displacements parallel to the direction of polarisation of the EM field. It can also be seen from Figs. 2 and 3 that in addition to a stable equilibrium at $x_{0}=y_{0}=0$ an unstable equilibrium is present at $x_{0}=y_{0} \approx 15$. The average RMS errors between the two models of the 16 data points calculated in Fig. 2 and Fig. 3 are $3.5 \%$ and $3.1 \%$, respectively. It is thus concluded that there is good agreement between the Barton treatment and the "exact" treatment, which can be expected for an almost paraxial Gaussian beam. However in a standard laser tweezers set up, the numerical aperture of the focusing lens is typically $N A>1$. Fig. 4 compares the restoring acceleration $\left[a_{z} / g\right]$ exerted on a spherical water droplet of diameter $2 \mu \mathrm{m}$ along the axis of propagation for an order 01 beam of wavelength $(\lambda=0.5145 \mu \mathrm{m})$ focused by $\mathrm{NA} \approx 1$ lens, which corresponds to a value of $k d=4$. The beam spot size in this case is $w_{0}=0.231 \mu \mathrm{m}$ and the beam power is $P=3.5 \mathrm{~mW}$. The offset along the $x$ and $y$ axis is zero $\left(x_{0}=y_{0}=0\right)$. As Barton et al. [5] have not presented these results, the results for the 5th order Gaussian beam approximation are calculated using the code of Ulanowski and Jones [13]. 


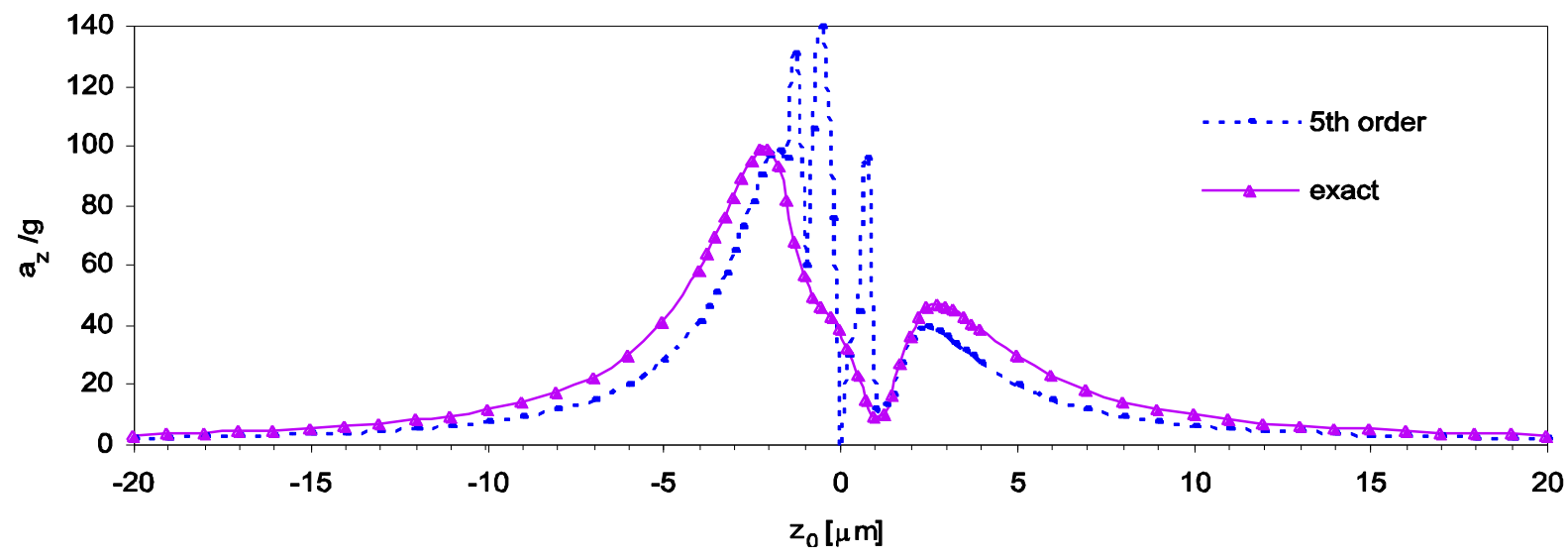

Fig. 4. Comparison of the restoring acceleration based on the 5th order approximation (doted curve) and the "exact" treatment (solid curve), along the propagation axis for optical levitation of a water droplet in air, using a focused, linearly polarised laser beam, $D=2 \mu \mathrm{m}, \bar{n}=1.334$, $\lambda_{0}=0.5145 \mu \mathrm{m}, w_{0}=0.231 \mu \mathrm{m}$, and $P=3.5 \mathrm{~mW}, k d=4$.

It can be seen from Fig. 4 that the results calculated using the 5th order Gaussian beam approximation (doted curve), are highly inaccurate in the range $z_{0}=[-1.25 \ldots 0.75]$. This is a clear indication that the 5 th order Gaussian beam approximation breaks down for strongly focused beams. Figs. 5 and 6 show a comparison of normalized (at the origin) irradiance profiles for the "exact" treatment versus the 5th order Gaussian beam approximation for $k d=4$, at the beam waist and at $z=0.75 \mu \mathrm{m}$ respectively. The breakdown of the 5th order Gaussian beam approximation for $k d=4$ can be clearly seen from the difference in the irradiance profiles of the two beams at $z=0.75 \mu \mathrm{m}$. Since the sphere has a diameter of $2 \mu \mathrm{m}$ it is apparent that even though the irradiance profiles are almost identical at the beam waist, the contributions from the differences at $z=0.75 \mu \mathrm{m}$ will have an effect on the trapping forces at $z_{0}=0$.

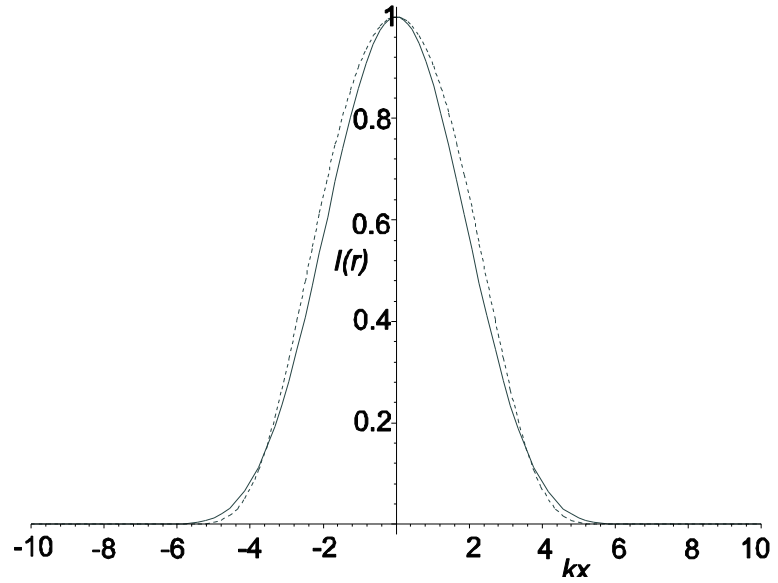

Fig. 5. Normalised irradiance profile at the beam waist for $k d=4$ : "exact" treatment (solid curve) versus 5th order Gaussian approximation (doted curve).

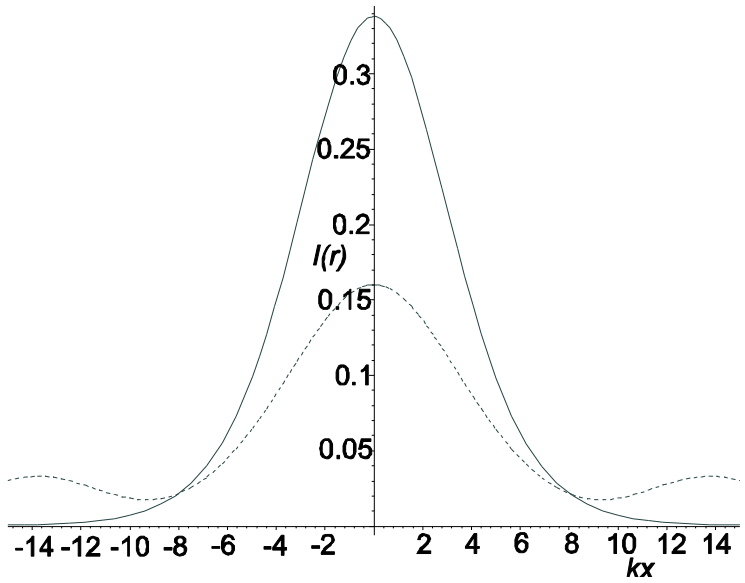

Fig. 6. Normalised irradiance profile at $z=0.75 \mu \mathrm{m}$ for $k d=4$ : "exact" treatment (solid curve) versus 5th order Gaussian beam approximation (doted curve). 
Furthermore, it can be seen from Fig. 4 that the restoring acceleration $\left[a_{z} / g\right]$ is always greater than 1 . Thus it can be concluded that no trapping occurs in this laser tweezers set up. The average RMS error between the two models of the 65 calculated data points in Fig. 4 is $44 \%$. The largest RMS error is for a sphere location at $z_{0}=0.75 \mu \mathrm{m}$, where the RMS error is $549.7 \%$. This result is not surprising, since Barton [7] claims that for an $s$ value of 0.3 , which corresponds to $k d=5.56$ the maximum error between the 5th order approximation and an exact solution to Maxwell's equation is $22.2 \%$ and for $s=0.4$, which corresponds to $k d=3.13$ it is $36.6 \%$. The reason for the discrepancy between the values given by Barton [7] and our values is, that the errors were calculated for 216 spatial positions, which not all correspond to the positions chosen by us.

In order to trap biological material it has proven useful [17] to use polystyrene spheres as "handles", since they can be more refractile than the biological substance, thus supplying extra trapping forces. Furthermore the shape and the uniform size facilitates the calibration of the laser tweezers. For this reason, the restoring acceleration induced onto a polystyrene $(n=1.6)$ sphere of diameter $2 \mu \mathrm{m}$ suspended in water is calculated for $k d=7.08$, a beam power $P=3.5 \mathrm{~mW}$, beam waist radius $w_{0}=0.231 \mu \mathrm{m}$ and wavelength of $\lambda_{0}=514.5 \mathrm{~nm}$. Polystyrene has a density $\rho=1.05 \times 10^{3} \mathrm{kgm}^{-3}$, giving sphere mass $m_{p}=4.4 \times 10^{-15} \mathrm{~kg}$. Fig. 7 compares the restoring acceleration for sphere positions along the axis of propagation $\left[a_{z} / g\right]$ calculated using the "exact" treatment and the 5th order Gaussian beam approximation model [13]. The offset along the $x$ and $y$ axis is zero $\left(x_{0}=y_{0}=0\right)$.

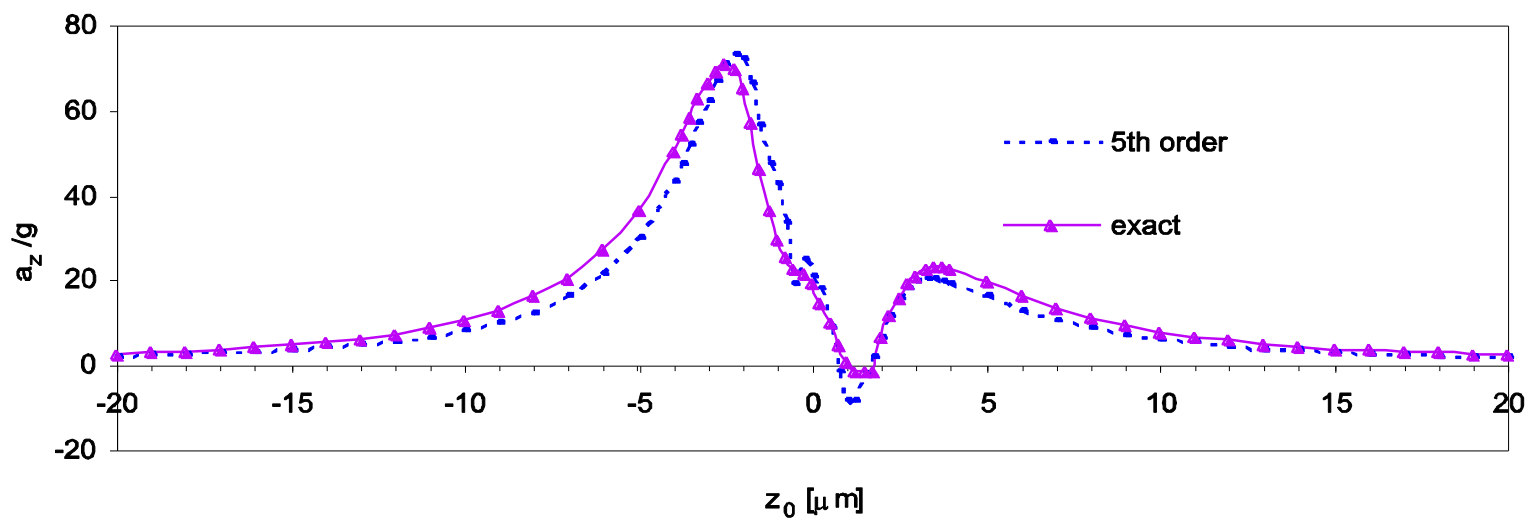

Fig. 7. Comparison of the restoring acceleration based on the 5th order Gaussian beam approximation

(doted curve) and the "exact" treatment (solid curve), along the propagation axis for optical trapping of a polystyrene sphere in water, using a focused, linearly polarised laser beam, $D=2 \mu \mathrm{m}, \lambda_{0}=0.5145 \mu \mathrm{m}$, $w_{0}=0.231 \mu \mathrm{m}, P=3.5 \mathrm{~mW}, k d=7.08$.

The average RMS error between the two models of the 65 data points is $48 \%$. The largest RMS error is for a sphere location at $z_{0}=1$, where the RMS error is $1248 \%$. From Fig. 7 it is seen that in the case of trapping a polystyrene sphere suspended in water, the difference between the two models is not as big as in the case of water droplet suspended in air. By comparing Fig. 7 with Fig. 4 it is apparent, that a polystyrene sphere suspended in water is subjected to a smaller restoring acceleration, than a water droplet suspended in air. It can be seen from Fig. 7 that the polystyrene sphere in water is subjected to maximum acceleration $a_{z} / g \approx 71$ at sphere location $x_{0}=y_{0}=0$ and $z_{0}=-2.5 \mu \mathrm{m}$. According to Fig. 4, the water droplet in air is subjected to a maximum acceleration $a_{z} / g \approx 98$ at sphere location $x_{0}=y_{0}=0$ and $z_{0}=-2.25 \mu \mathrm{m}$. Thus the polystyrene sphere in water is accelerated 1.4 times less than the water droplet in air. One of the reasons for the lower acceleration value is that the beam is less strongly focused. The other reason can be understood qualitatively from geometrical optics. The relative refractive index for the air water interface is $\bar{n}=1.334$ and the relative refractive index of the polystyrene water interface 
is $\bar{n}=1.2$. Hence the relative refractive index for the polystyrene water interface is lower than the relative refractive index for the air water interface. It follows that a polystyrene sphere in water reflects less radiation than a water droplet in air and so the magnitude of the forward directed force is lower. At the same time, the magnitude of the forward directed force relative to the backward directed force can become lower for a polystyrene sphere in water than for a water droplet in air, allowing stable trapping.

In order to calculate the restoring acceleration along the polarization axis and perpendicular to the polarization axis, the vertical position of the polystyrene sphere is $z_{0}=1.25 \mu \mathrm{m}$, as at this point the restoring acceleration along the axis of propagation $\left[a_{z} / g\right]$ has according to Fig. 7 a minimum. Fig. 8 shows the restoring acceleration along the axis of polarization and Fig. 9 represents the restoring acceleration perpendicular to the polarization axis.

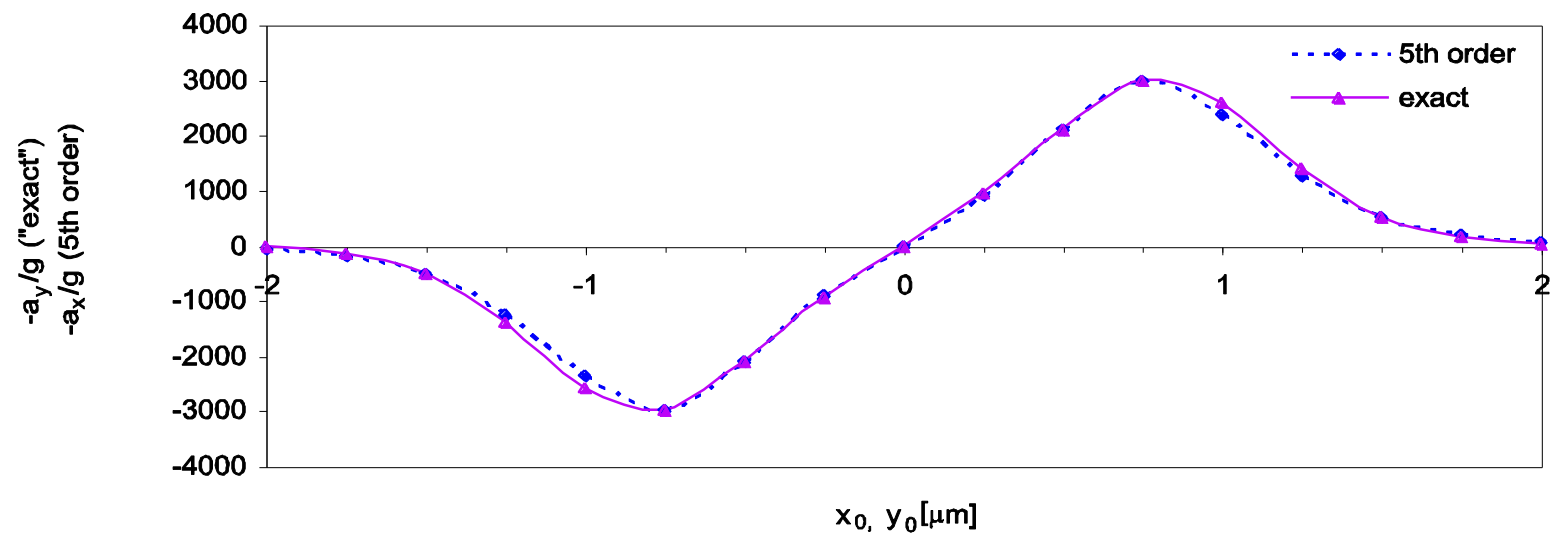

Fig. 8. Comparison of the restoring acceleration based on the 5th order Gaussian beam approximation (doted curve) and the "exact" treatment (solid curve), along the polarisation axis versus displacement along the polarisation axis for optical trapping of a polystyrene sphere in water, using a focused order 01 mode linearly polarised laser beam $\left(x_{0}=y_{0}=0, z_{0}=1.25 \mu \mathrm{m}\right.$, $\overline{\mathrm{n}}=1.2, D=2 \mu \mathrm{m}, \lambda_{0}=0.5145 \mu \mathrm{m}, w_{0}=0.231 \mu \mathrm{m}, k d=7.08$ and $\left.P=3.5 \mathrm{~mW}\right)$. 


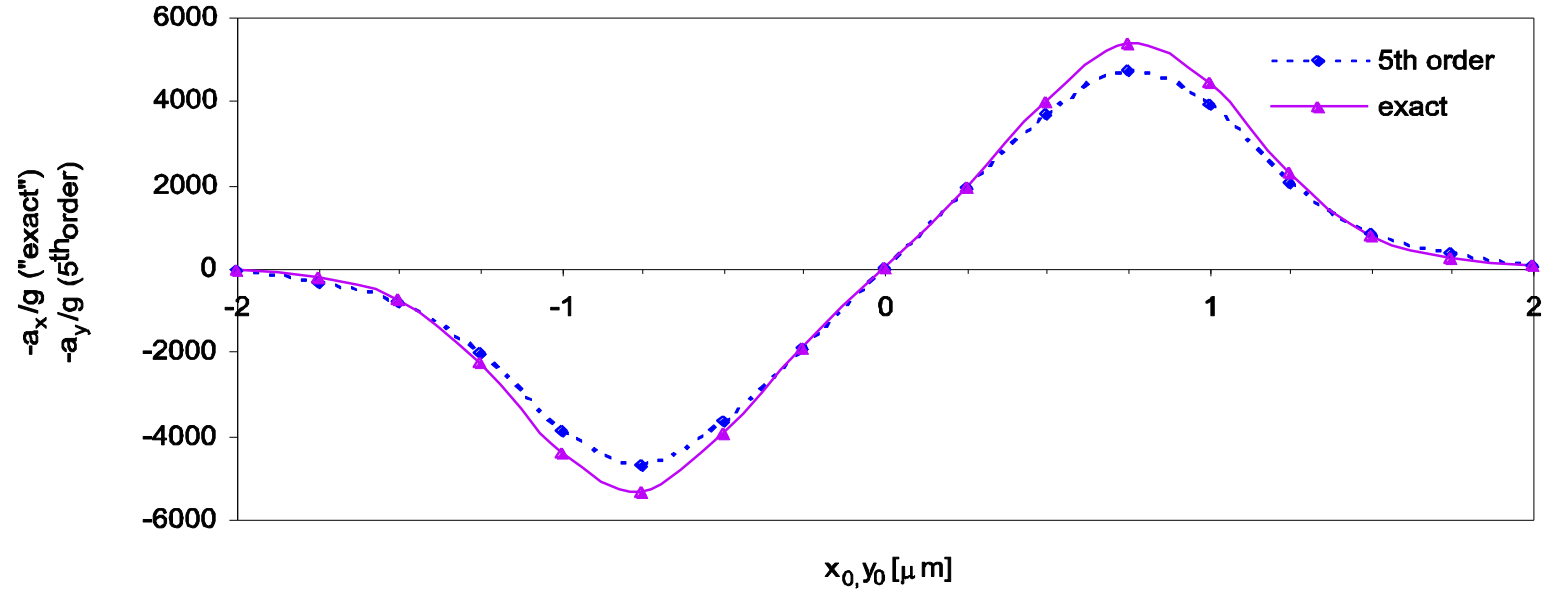

Fig. 9. Comparison of the trapping efficiency based on the 5th order Gaussian beam approximation (doted curve) and the "exact" treatment (solid curve), along the axis perpendicular to the polarisation axis versus displacement along the axis perpendicular to the polarisation axis for optical trapping of a polystyrene sphere in water, using a focused order 01 mode linearly polarised laser beam $\left(x_{0}=y_{0}=0\right.$, $z_{0}=1.25 \mu \mathrm{m}, \overline{\mathrm{n}}=1.2, D=2 \mu \mathrm{m}, \lambda_{0}=0.5145 \mu \mathrm{m}, w_{0}=0.231 \mu \mathrm{m}, k d=7.08$ and $\left.P=3.5 \mathrm{~mW}\right)$.

The average RMS errors between the two models of the 17 data points in Figs. 8 and 9 are $13 \%$ and $19 \%$ respectively. According to Figs. 8 and 9 the restoring acceleration along the polarisation axis of the beam is, as expected, greater than the restoring acceleration along the axis perpendicular to the direction of polarisation. Hence, even though the polarization dependence of the Fresnel coefficients is given for plane EM waves, it can be concluded that it is also applicable for the EM fields of a strongly focused Gaussian beams.

\section{Discussion}

An electromagnetic field, which represents an arbitrarily focused Gaussian laser beam has been presented. This field is linearly polarized along the $y$ axis in the paraxial limit and also satisfies the Richards and Wolf [16] far-field boundary conditions. Optical trapping forces have been calculated using the method presented by Barton et al. [5], which is valid for arbitrary EM fields. Figs. 1, 2 and 3 shows that the trapping forces calculated using the 5th order approximation are accurate for a nearly paraxial Gaussian beam (NA = 0.246), when compared to the exact treatment. The average RMS errors between the two models of the calculated data points in these cases are $1.4 \%, 3.53 \%$ and $3.13 \%$ respectively. However, for strongly focused beams, $k d=4$, the 5 th order approximation becomes unreliable: we observe in Fig. 4 a breakdown of the approximation when trapping a water droplet in air near the focal point $\left(z_{0}=[-1.25 \ldots 0.75]\right)$. From Figs 7,8 and 9 it can be seen that when trapping a polystyrene sphere in water the 5th order approximation does not break down completely, but the average RMS errors between the two models of the calculated data points are $48 \%, 13 \%$ and $19 \%$ respectively. When comparing these results to the ones obtained by Moine and Stout [6] it can be concluded that they are of the same order of magnitude. However the calculations presented by [6] are for $k d=4.93$ and for $\bar{n}=1.09$, where here $k d=7.08$ and $\bar{n}=1.2$. As their value of $k d$ is larger than the one used in Fig. 4 and their value of the relative refractive index is lower than the one used in the figure presented here, it is not surprising that 
Moine and Stout [6] did not observe a break down of the 5th order approximation or the small oscillations near $z=0$. It has thus been established that it is important to have a focused laser beam description that is accurate under non-paraxial conditions, since in order to trap a particle effectively the laser beam needs to be strongly focused. Furthermore it has been shown that the polarization dependence of the Fresnel coefficients also applies for strongly focused Gaussian beams, despite the fact that they are derived for plane EM waves.

\section{References}

[1] Ashkin A. Acceleration and trapping of particles by radiation pressure. Phys Rev Lett 1970;24:156-159.

[2] Tadir $\mathrm{Y}$, Wright $\mathrm{WH}$, Vafa $\mathrm{O}$ et al. Micromanipulation of sperm by laser Generated optical trap. Fertil Steril 1989;52:870-873.

[3] Block SM, Blair DF, Berg HC. Compliance of bacterial flagella measured with optical tweezers. Nature 1989;338:514-518.

[4] Ashkin A, Schütze K, Dziedzic JM, Euteneuer U, Schliwa M. Forces generated of organelle transport measured in vivo by an infrared laser trap. Nature 1990;348:346-348.

[5] Barton JP, Alexander DR, Schaub SA. Theoretical determination of net radiation forces and torque for a spherical particle illuminated by a focused laser beam. J Appl Phys 1989;66:45944602.

[6] Moine O,Stout B. Optical force calculations in arbitrary beams by use of the vector addition theroem. J Opt Soc Am B 2005;22:1620-1631.

[7] Barton JP,Alexander DR. Fifth-order corrected EM field components for a fundamental Gaussian beam. J Appl Phys 1989;66:2800-2802.

[8] Dorizzi RR. Computation of Forces exerted on a Microparticle by a Laser Beam. PhD thesis, University of Hertfordshire 2004.

[9] Gouesbet G, Gréhan G, Maheu B. Localized interpretation to compute all the coefficients $g_{n}{ }^{m}$ in the generalized Lorenz-Mie theory. J Opt Soc Am A 1990;7:998-1007.

[10] Mazolli A, Maia Neto PA, Nussenzveig HM. Theory of trapping forces in optical tweezers. Proc R Soc Lond A 2003;459:3021-3041.

[11] Lorenz L. Sur la lumière réfléchie et réfractée par une sphère transparente. in Oeuvres

Scientifiques de L. Lorenz, revues et annotées par H. Valentiner (Librairie Lehmann et Stage, Copenhagen 1898).

[12] Mie G. Beiträge zur Optik trüber Medien, speziell kolloidaler Metal-Lösungen. Ann Phys 1908; 25:377-445. 
[13] Jones NT. Modelling laser entrapment forces on microspheres. MSc project report, University of Hertfordshire 1994.

[14] Bohren CF, Huffman DR. Absorption and Scattering of Light by Small Particles. WileyInterscience 1983.

[15] Ulanowski Z, Ludlow LK. Scalar field of non-paraxial Gaussian beams. Opt Lett 2000;25:17921794.

[16] Richards B, Wolf E. Electromagnetic diffraction in optical systems, II. Structure of the image field in an aplanatic system. Proc R Soc London Ser A 1959;253:358-379.

[17] Svoboda K, Block SM. Biological Applications of optical forces. Ann Rev Biophys Biomol Struct 1994;247-285. 